\title{
Increased oncogenic microRNA-18a expression in the peripheral blood of patients with prostate cancer: A potential novel non-invasive biomarker
}

\author{
GHADAAL-KAFAJI $^{1}$, ZIAD TARIQ AL-NAIEB ${ }^{2}$ and MOIZ BAKHIET ${ }^{1}$ \\ Departments of ${ }^{1}$ Molecular Medicine and ${ }^{2}$ Surgery, College of Medicine and Medical Sciences, \\ Arabian Gulf University, Manama 329, Kingdom of Bahrain
}

Received November 30, 2014; Accepted November 10, 2015

DOI: $10.3892 / \mathrm{ol} .2015 .4014$

\begin{abstract}
MicroRNAs have been demonstrated to be stably detectable in peripheral blood, thus representing important sources of non-invasive biomarkers of various diseases, including cancer. Recently, microRNA-18a (miR-18a) has been revealed to be highly expressed in prostate cancer (PC) tissues, acting as an oncogenic miRNA. The present study evaluated miR-18a expression in the peripheral blood of patients with PC, patients with benign prostatic hyperplasia (BPH), and healthy individuals, to assess the feasibility of using peripheral blood miR-18a as a potential non-invasive biomarker for PC. Total RNA was extracted from peripheral whole blood samples from $24 \mathrm{PC}$ patients, $24 \mathrm{BPH}$ patients and 23 healthy control individuals. The expression of miR-18a was assessed by reverse transcription quantitative polymerase chain reaction. The results revealed that miR-18a expression was significantly higher in PC patients than in BPH patients and healthy controls [fold change (mean \pm standard deviation), 5.5 \pm 1.4 for PC, $1.5 \pm 0.5$ for $\mathrm{BPH}$ and $1.2 \pm 0.6$ for controls; $\mathrm{P}<0.005]$. Higher miR-18a expression was strongly associated with PC [odds ratio (OR), 4.602; 95\% confidence interval (CI), 2.194-9.654; $\mathrm{P}=0.001]$, but was not significantly associated with $\mathrm{BPH}$ (OR, 1.2; 95\% CI, 0.7-2.02; $\mathrm{P}=0.332$ ). Despite the small number of patients, which limits the statistical power of the study, higher miR-18a expression was observed to be significantly correlated with certain clinicopathological parameters, including Gleason score $>7$ and pathological tumor stage $3 / 4(\mathrm{P}<0.005)$. A receiver operating characteristic (ROC) analysis revealed that miR-18a discriminated $\mathrm{PC}$ patients from $\mathrm{BPH}$ patients and healthy controls [area under the curve (AUC), 0.805; 95\% CI,
\end{abstract}

Correspondence to: $\mathrm{Dr}$ Ghada Al-Kafaji, Department of Molecular Medicine, College of Medicine and Medical Sciences, Arabian Gulf University, Salmanyia Avenue, Building 293, Road 2904, Manama 329, Kingdom of Bahrain

E-mail: ghadaa@agu.edu.bh

Key words: prostate cancer, benign prostatic hyperplasia, microRNA, biomarker, peripheral blood miR-18
0.704-0.906). Furthermore, use of the ROC curve to discriminate PC from BPH patients yielded an AUC of $0.878(95 \%$ CI, 0.783-0.972). In summary, the present results indicate that miR-18a expression is significantly increased in peripheral blood of patients with PC compared with that of BPH patients and healthy individuals, and that higher miR-18a expression is associated with progression of PC. Peripheral blood oncogenic miR-18a may serve as a potential novel non-invasive biomarker for $\mathrm{PC}$ that also facilitates discrimination between $\mathrm{PC}$ and $\mathrm{BPH}$.

\section{Introduction}

Prostate cancer (PC) is the most widely diagnosed malignant tumor and the second leading cause of cancer-associated mortality in males (1). The mechanisms underlying the occurrence and progression of the disease remain largely unknown. Generally, patients with PC do not exhibit symptoms until the disease becomes locally advanced or metastatic (2). Prostate-specific antigen (PSA) detection in serum is the current standard diagnostic tool for PC. In healthy males, PSA is present in small quantities in the serum (typically $<4 \mathrm{ng} / \mathrm{ml}$ ), while those with PC generally present with a higher PSA (3). However, serum PSA has been demonstrated to be elevated in various other conditions, including urinary retention and prostatitis, and following trauma or physical manipulation (3). Furthermore, serum PSA may be increased in patients with benign prostate hyperplasia $(\mathrm{BPH})$, which often leads to unnecessary biopsies (4). The development of more effective non-invasive biomarkers would be clinically significant for the detection of PC, and also to facilitate the differentiation of PC from BPH $(4,5)$.

MicroRNAs (miRNAs) are short non-coding RNA molecules with an average length of 22 nucleotides. They regulate gene expression through binding to target messenger RNAs (mRNAs) and promoting their degradation and/or directing post-transcriptional repression (6). miRNAs are important for a wide range of cellular functions, including cell proliferation, differentiation and apoptosis (7). Aberrant expression of miRNAs is also implicated in various diseases, including cancer (8). Analysis of miRNA expression profiles in cancer cells has revealed that dysregulation of these molecules 
contributes to the aberrant activation of oncogenes and the inactivation of tumor suppressor genes in human carcinogenesis (9), and several of these miRNAs have been reported to be both up- or downregulated $(10,11)$. In particular, miRNA (miR)-18a, which is located in the potentially oncogenic miR-17-92 cluster $(12,13)$, is highly expressed in various types of cancer (13-15). Furthermore, miR-18a has recently been demonstrated to act as an oncogenic miRNA in PC by promoting tumorigenesis in in vitro and in vivo models of PC (16).

It has been demonstrated that miRNAs are present in human peripheral blood and exhibit resistance to endogenous RNase activity (17). In addition, miRNAs circulate in the blood of patients with diseases and of healthy individuals, and are remarkably stable, making their isolation and analysis easy (17). These unique characteristics make peripheral blood miRNAs potential novel sources of non-invasive biomarkers.

Although the origin and function of blood miRNAs are unclear, it has been demonstrated that miRNAs may be packaged into lipid-based carriers, such as exosomes, microparticles or apoptotic bodies, and actively secreted into the circulation where they bind to neighboring cells to regulate the expression of target genes, thus mediating cell-to-cell communication (18).

To date, circulating miRNAs in serum and plasma have been investigated as potential diagnostic and prognostic biomarkers for a diverse range of diseases (19-21). A number of studies have also revealed that miRNAs circulating in whole blood serve as a novel class of biomarkers in various types of malignancy, including brain (22) and breast (23) cancers, as well as in non-malignant diseases $(24,25)$. Furthermore, a recent study by Median-Villaamil et al (26) has demonstrated that specific miRNAs are differently expressed in the whole blood of PC patients compared with that in healthy individuals, suggesting their potential for use as biomarkers in the diagnosis of PC.

The aim of the present study was to evaluate miR-18a expression in the peripheral whole blood of PC and $\mathrm{BPH}$ patients, and in healthy individuals, in order to test the potential of blood miR-18a as non-invasive biomarker for PC.

\section{Materials and methods}

Participants. The current study was approved by the Medical Research and Ethics Committee of the College of Medicine and Medical Sciences, Arabian Gulf University (Manama, Bahrain). Between June 2013 and January 2014, whole blood samples were collected at the Urological Clinic of King Abdullah Medical City (Manama, Bahrain). The samples were obtained from 24 patients with surgically treated, localized PC, one day prior to radical prostatectomy. Tumor aggressiveness was determined by histological tumor grading system in the Gleason grading $(27,28)$. Prostatic carcinomas with final score $<7$ were considered low-grade, and with a final score $>7$ were considered high-grade. PC was staged using the TNM system, which assesses the tumor (T), lymph nodes (N) and metastases (M) (29). Blood samples were also obtained from 24 patients diagnosed with BPH by biopsy specimen of the prostate and who exhibited an increased PSA level ( $>4 \mathrm{ng} / \mathrm{ml})$ or abnormal digital rectal examination findings, and from 23 healthy controls. All participants provided written informed consent prior to blood collection. As treatment may influence the level of circulating miRNAs, only blood samples from patients who received no hormone ablative or cytotoxic therapy were used.

RNA extraction. Whole blood samples $(5 \mathrm{ml})$ were collected in tubes containing EDTA. Aliquots $(0.5 \mathrm{ml}$ each) of EDTA blood were mixed with $1.3 \mathrm{ml}$ RNAlater ${ }^{\circledR}$, an RNA stabilization reagent (Ambion; Thermo Fisher Scientific, Austin, TX, USA). Samples were stored at $-80^{\circ} \mathrm{C}$ until RNA extraction. Prior to RNA extraction, frozen samples were thawed at room temperature, and total RNA (including small RNA) was isolated using the miRNeasy kit (Qiagen, Inc., Valencia, CA, USA) according to the manufacturer's recommendations. Concentration and purity of RNA was quantified by measuring the absorbance at $260 \mathrm{~nm}\left(\mathrm{~A}_{260}\right)$ and $280 \mathrm{~nm}\left(\mathrm{~A}_{280}\right)$ using a Thermo Scientific ${ }^{\mathrm{TM}}$ Nanodrop ND-100 spectrophotometer (Thermo Fisher Scientific, Waltham, MA, USA).

Reverse transcription $(R T)$ and quantitative $(q)$ polymerase chain reaction $(P C R)$. For $\mathrm{RT}$, an Applied Biosystems ${ }^{\mathrm{TM}}$ TaqMan MicroRNA Reverse Transcription kit (Thermo Fisher Scientific) was used. Total RNA (20 ng) was mixed with specific stem-loop RT primers $(3 \mu \mathrm{l}), 100 \mathrm{mM}$ dNTPs $(0.15 \mu \mathrm{l}), 10 \mathrm{X}$ RT buffer $(1.5 \mu \mathrm{l}), 20 \mathrm{U} / \mu \mathrm{l}$ RNase inhibitor $(0.19 \mu \mathrm{l}), 50 \mathrm{U} / \mathrm{ml}$ MultiScribe ${ }^{\mathrm{TM}}$ Reverse Transcriptase $(1 \mu \mathrm{l})$ and nuclease-free water $(4.16 \mu \mathrm{l})$ to a final volume of $15 \mu \mathrm{l}$. For synthesis of cDNA, reaction mixtures were incubated at $16^{\circ} \mathrm{C}$ for $30 \mathrm{~min}$, at $42^{\circ} \mathrm{C}$ for $30 \mathrm{~min}$ and at $85^{\circ} \mathrm{C}$ for $5 \mathrm{~min}$, and then held at $4^{\circ} \mathrm{C}$. Subsequently, $1.33 \mu \mathrm{l}$ of cDNA was amplified using $10 \mu \mathrm{l}$ of TaqMan 2X Universal PCR Master Mix II (Applied Biosystems; Thermo Fisher Scientific), $1 \mu 1$ of gene-specific primers and $7.67 \mu \mathrm{l}$ of nuclease-free water in a final volume of $20 \mu \mathrm{l}$. qPCR was run on a 7900HT Real-Time PCR system (Applied Biosystems; Thermo Fisher Scientific), and the reaction mixtures were incubated at $95^{\circ} \mathrm{C}$ for $10 \mathrm{~min}$, followed by 40 cycles of $95^{\circ} \mathrm{C}$ for $15 \mathrm{sec}$ and $60^{\circ} \mathrm{C}$ for $1 \mathrm{~min}$. The PCR primers had the following sequences: Mature hsa-miR-18a (target), 5'-UAAGGUGCAUCUAGUGCAGAU AG-3'; and RNU6B (reference), 5'-CGCAAGGATGACACG CAAATTCGTGAGCGTTCCATATTTTT-3'.

The threshold cycle $(\mathrm{Ct})$ values were calculated using SDS software version 1.4 (Applied Biosystems; Thermo Fisher Scientific). The expression of miR-18a in the blood was normalized to the expression of U6 small nuclear RNA (RNU6B). The fold change in miR-18a expression was determined using the $2^{-\Delta \Delta \mathrm{Ct}}$ method (30). $\Delta \mathrm{Ct}$ was calculated by subtracting the $\mathrm{Ct}$ values of RNU6B from the $\mathrm{Ct}$ values of the miR-18a. $\Delta \Delta \mathrm{Ct}$ was then calculated by subtracting the $\Delta \mathrm{Ct}$ of the healthy control samples from the $\Delta \mathrm{Ct}$ of the case samples (PC and $\mathrm{BPH}$ ).

Statistical analysis. Statistical analyses were performed using SPSS software version 19.0 (IBM SPSS, Armonk, NY, USA). Comparisons between cases and controls were analyzed by $\chi^{2}$ or Student's $t$-tests, and data were presented as the mean \pm standard deviation (SD). Multivariate logistic regression analysis was used to assess the association of miR-18a expression with $\mathrm{PC}$ and $\mathrm{BPH}$. Receiver operating characteristic (ROC) curves were used to assess miR-18a as a biomarker, and the area under the curve (AUC) was reported. $\mathrm{P}<0.05$ (two-tailed) was considered to indicate a statistically significant difference. 
Table I. Characteristics of participants.

\begin{tabular}{|c|c|c|c|}
\hline Variable & Prostate cancer & Benign prostatic hyperplasia & Healthy controls \\
\hline Total subjects; $\mathrm{n}$ & 24 & 24 & 23 \\
\hline Age, years; mean (range) & $71(65-79)$ & $70(66-78)$ & $71(65-76)$ \\
\hline Prostate-specific antigen, ng/ml; mean $( \pm \mathrm{SD})$ & $17.8( \pm 4.72)$ & $10.5( \pm 2.65)$ & $3.5( \pm 0.40)$ \\
\hline \multicolumn{4}{|l|}{ Gleason score; n (\%) } \\
\hline$<7$ (low) & $5(20.8)$ & - & - \\
\hline 7 (intermediate) & $10(41.7)$ & - & - \\
\hline$>7$ (high) & $9(37.5)$ & - & - \\
\hline \multicolumn{4}{|l|}{ Tumor stage; n (\%) } \\
\hline $\mathrm{pT} 1 / 2$ & $14(58.3)$ & - & - \\
\hline pT3/4 & $10(41.7)$ & - & - \\
\hline \multicolumn{4}{|l|}{ Node stage; n (\%) } \\
\hline N0 & $22(91.6)$ & - & - \\
\hline N1 & $2(8.3)$ & - & - \\
\hline \multicolumn{4}{|l|}{ Metastasis stage; $\mathrm{n}(\%)$} \\
\hline M0 & $24(100.0)$ & - & - \\
\hline M1 & $0(0.0)$ & - & - \\
\hline
\end{tabular}

\section{Results}

Patient characteristics. The demographics and clinical characteristics of the participants are outlined in Table I. No significant differences in age were observed between PC patients, BPH patients and healthy control individuals $(\mathrm{P}>0.005)$. Serum PSA levels were significantly higher in PC patients than in healthy control individuals $(\mathrm{P}<0.005)$, and were significantly increased in $\mathrm{BPH}$ patients compared with that of healthy controls $(\mathrm{P}<0.005)$.

miR-18a expression in PC, BPH and healthy controls. The expression of miR-18a was determined relative to the endogenous control, RNU6B, in the peripheral blood of PC and BPH patients and healthy control subjects using qPCR. Values were expressed as the mean $( \pm \mathrm{SD})$ fold difference in gene expression. miR-18a and RNU6B yielded reliable $\mathrm{Ct}$ values in all samples from PC, BPH and control subjects. No replicates with $\mathrm{Ct}>35$ were detected.

The expression of miR-18a normalized to that of RNU6B was significantly increased in the peripheral blood of patients with PC compared with that of BPH patients and healthy control individuals $(\mathrm{P}<0.005)$ (Fig. 1). The mean relative expression of miR-18a was $5.5 \pm 1.4$ for PC patients, $1.5 \pm 0.5$ for BPH patients and $1.2 \pm 0.6$ for healthy controls. Multivariate logistic regression analysis revealed a strong association between higher miR-18a expression and PC [odds ratio (OR), 4.602; 95\% confidence interval $(\mathrm{CI}), 2.194-9.654 ; \mathrm{P}=0.001)$. By contrast, higher miR-18a was not significantly associated with BPH (OR, 1.2; 95\% CI, 0.7-2.02; $\mathrm{P}=0.332$ ).

Association between miR-18a expression and pathological variables. PC patients with more aggressive tumors exhibited significantly higher miR-18a expression compared with that of patients with less aggressive tumors. The mean relative

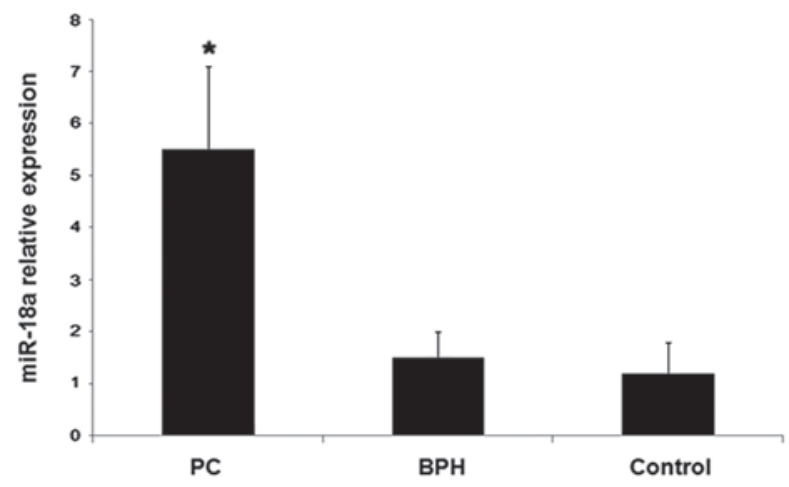

Figure 1. miR-18a expression in peripheral blood of PC patients, BPH patients and healthy individuals. The expression of miR-18a relative to that of U6 small nuclear RNA was determined by quantitative polymerase chain reaction in peripheral blood samples from PC patients $(n=24), B P H$ patients $(n=24)$ and healthy control individuals $(n=23)$. Data are presented as the mean \pm standard deviation. ${ }^{*} \mathrm{P}<0.05$ vs. $\mathrm{BPH}$ patients and controls. miR-18a, microRNA-18a; PC, prostate cancer; $\mathrm{BPH}$, benign prostatic hyperplasia.

miR-18a expression in the group of patients with a Gleason score $<7$ was $2.4 \pm 0.4$, and increased progressively to $4.4 \pm 0.3$ in patients with a Gleason score of 7, and to 8.6 40.8 in patients with a Gleason score $>7$ (Fig. 2A). In addition, miR-18a expression was significantly higher in patients at an advanced stage of disease: Patients with a pathological tumor (pT) stage of 3 or 4 exhibited a mean relative miR-18a expression level of $8.2 \pm 1.4$, which was significantly increased compared with patients of pT stage 1 or 2 , in whom a mean relative expression level of 3.6 \pm 1.0 was observed $(\mathrm{P}<0.005)$ (Fig. 2B).

miR-18a expression as a diagnostic tool. As miR-18a was differentially expressed in the peripheral blood of PC patients, $\mathrm{BPH}$ patients and control subjects, the potential of peripheral blood oncogenic miR-18a as a biomarker was evaluated. Based 

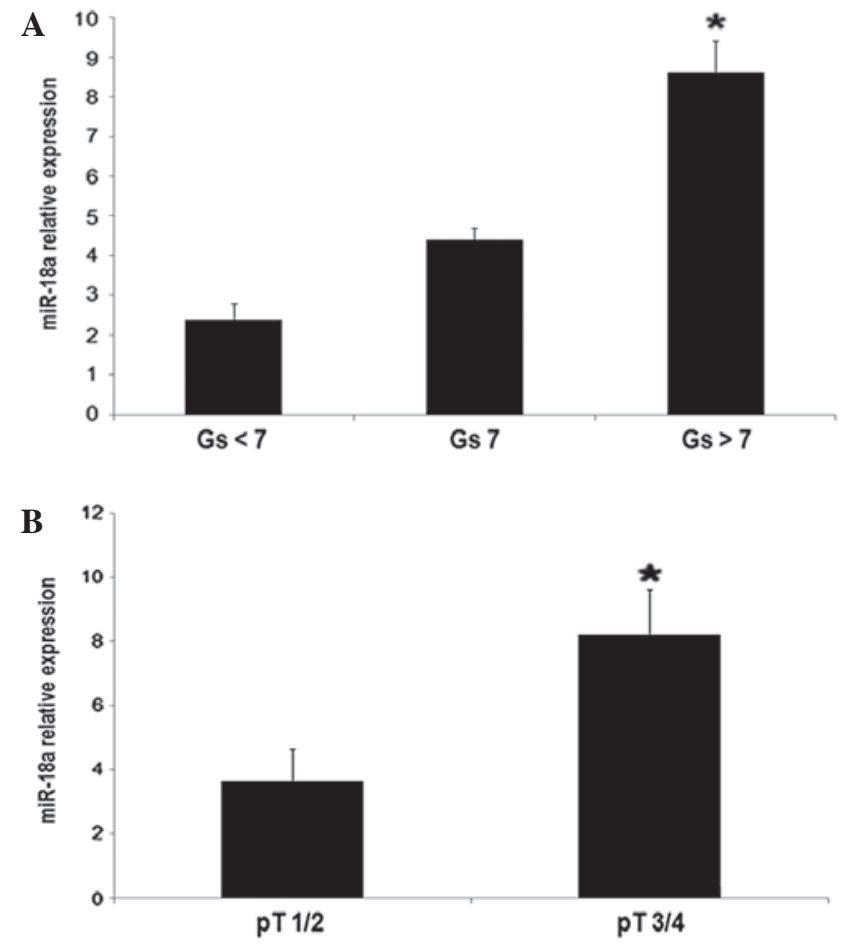

Figure 2. Association between pathological parameters and miR-18a expression in peripheral blood of prostate cancer patients. (A) Relative miR-18a expression in patients with $\mathrm{Gs}<7(\mathrm{n}=5), \mathrm{Gs}=7(\mathrm{n}=10)$ and $\mathrm{Gs}>7(\mathrm{n}=9)(\mathrm{P}<0.05$ vs. Gs $=7$ and $<7$ ). (B) Relative miR-18a expression in patients of stage $\mathrm{pT} 1 / 2$ $(\mathrm{n}=14)$ and $\mathrm{pT} 3 / 4(\mathrm{n}=10)(\mathrm{P}<0.05$ vs. $\mathrm{pT} 1 / 2)$. miR-18a expression was measured relative to U6 small nuclear RNA using quantitative polymerase chain reaction, and all data are presented as the mean \pm standard deviation. miR-18a, microRNA-18a; Gs, Gleason score; pT, pathological tumor stage.

on an ROC analysis, blood miR-18a was able to distinguish PC patients from BPH patients and controls (AUC, $0.805 ; 95 \% \mathrm{CI}$, 0.704-0.906; P<0.001; Fig. 3A). Furthermore, miR-18a was able to distinguish PC patients from BPH patients (AUC, 0.878; 95\% CI, 0.783-0.972; P<0.001; Fig. 3B).

\section{Discussion}

miRNAs are part of a class of small non-coding RNAs that function as translational repressors by partially pairing to the $3^{\prime}$ untranslated region of target mRNAs (7). miRNAs are involved in highly regulated processes, including proliferation, differentiation and apoptosis (7), and are also implicated in a range of diseases (8). Notably, miRNAs serve significant roles in cancer owing to their ability to function as regulators of tumor suppressors and oncogenes (9).

Circulating tumor-derived miRNAs were first described in peripheral blood by Mitchell et al (17), who found that circulating miRNAs had the potential to be novel biomarkers in patients with PC. The authors showed that tumor-derived miRNAs can enter the circulation even when originating from an epithelial cancer type. They also showed that circulating miRNAs in plasma remained stable even after prolonged room temperature incubation or cycles of freezing-thawing. Subsequently, circulating miRNAs in the serum, plasma and whole blood have been reported as biomarkers in various different types of cancer, in addition to other diseases (19-26).
A

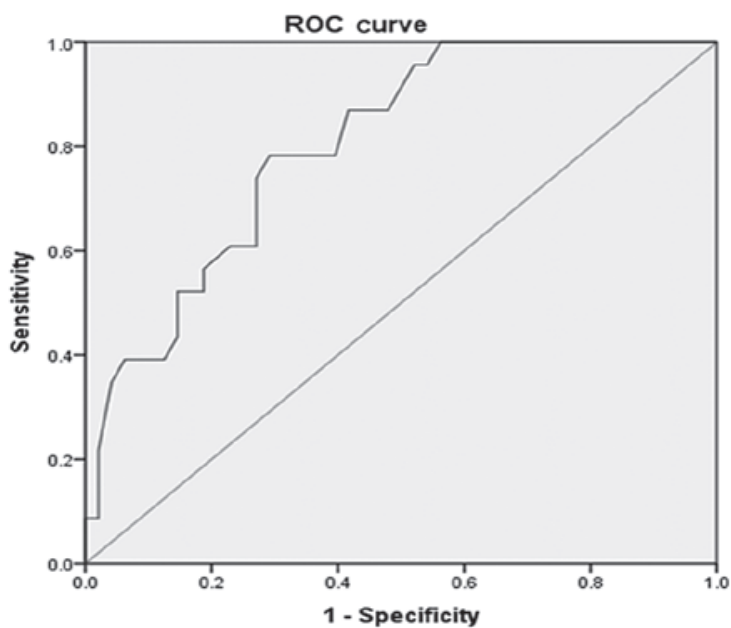

B

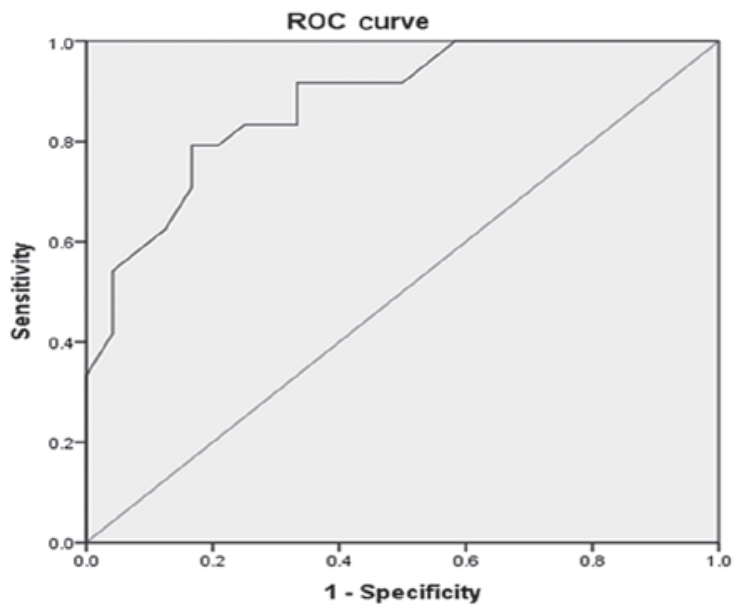

Figure 3. ROC curve analysis (A) miR-18a is able to distinguish PC patients from $\mathrm{BPH}$ patients and healthy control individuals (AUC, 0.864; 95\% CI, 0.751-0.977; $\mathrm{P}<0.001$ ). (B) miR-18a can distinguish $\mathrm{PC}$ patients from $\mathrm{BPH}$ patients (AUC, 0.878; 95\% CI, 0.783-0.972; $\mathrm{P}<0.001$ ). ROC, receiver operating characteristic; miR-18a, microRNA-18a; PC, prostate cancer; BPH, benign prostatic hyperplasia; AUC, area under the curve; CI, confidence interval.

The present study evaluated the levels of miR-18a, a highly expressed miRNA in the tissues of PC (16), in the peripheral blood of patients with PC or BPH, and in healthy individuals, to test the feasibility of using peripheral blood miR-18a as a potential novel non-invasive biomarker for PC.

miR-18a is expressed as part of the miR-17-92 cluster, which comprises seven miRNAs: miR-17-3p, miR-17-5p, miR-18a, miR-19a, miR-19b, miR-20a and miR-92a. This cluster has been reported to have potential oncogenic functions in tumorigenesis and cancer development $(12,13)$. In particular, miR-18a has been demonstrated to be significantly elevated in various types of cancer, including gastric cancer (14) and urothelial carcinomas of the bladder (15).

Recently, miR-18a has been reported to exhibit oncogenic activity in PC by targeting serine/threonine-protein kinase 4 (STK4) (16). Upregulation of miR-18a is capable of inducing a decrease in the STK4 protein level, and an increase in AKT phosphorylation to enhance tumor cell survival. By contrast, miR-18a knockdown in nude mice was demonstrated to decrease prostate tumor growth and induce apoptosis (16). STK4 is a proapoptotic protein that was identified as a tumor suppressor in cancer cells $(31,32)$. Therefore, inhibition of 
miR-18a expression was suggested as a therapeutically beneficial strategy for the treatment of PC (16).

The results of the current study demonstrated that the expression of peripheral blood miR-18a was significantly higher in patients with PC than in BPH patients and healthy control individuals. Furthermore, a strong association was observed between higher miR-18a expression and PC. In addition, significantly higher expression of blood miR-18a was detected in PC patients with more aggressive tumors (Gleason score $>7$ ) and of a more advanced stage (pT3/4), suggesting that miR-18a expression may correlate with progression of the disease.

Increased expression of circulating miR-18a has been previously reported in the plasma of patients with pancreatic cancer (33), and higher plasma miR-18 expression has also been observed in patients with colorectal cancer (34). Furthermore, circulating miR-18a has been demonstrated to be a promising biomarker for the diagnosis and monitoring of esophageal squamous cell carcinoma (35).

In the current study, an ROC analysis revealed that peripheral blood miR-18a was able to discriminate patients with PC from BPH patients and healthy controls. Notably, peripheral blood miR-18a was additionally able to differentiate between patients with $\mathrm{PC}$ and patients with $\mathrm{BPH}$.

$\mathrm{PC}$ is the most frequently diagnosed malignancy and the second major cause of cancer-associated mortality among males (1). BPH is commonly misdiagnosed as PC (36), and this frequently leads to unnecessary biopsies (3). The evaluation of non-invasive biomarkers in peripheral blood could aid in the early detection of PC, and also facilitate the discrimination between patients with malignant and benign prostatic tumors, thereby avoiding the need for invasive biopsies. Such biomarkers may also be important in the future treatment of this cancer.

A limitation of the present study is the small sample size, which may restrict the statistical power of the study. Therefore, the potential of peripheral blood miR-18a expression as a biomarker for PC must be confirmed and validated in larger populations.

In conclusion, the current results demonstrated that expression of miR-18a is increased in the peripheral blood of patients with PC compared with BPH patients and healthy controls, and that higher miR-18a expression is correlated with PC progression. Thus, peripheral blood oncogenic miR-18a may offer potential for utilization as a novel non-invasive biomarker for $\mathrm{PC}$ that also allows discrimination between $\mathrm{PC}$ and $\mathrm{BPH}$.

\section{Acknowledgements}

This work was supported by a research grant from the College of Medicine and Medical Sciences at Arabian Gulf University (grant no. 93).

\section{References}

1. Jemal A, Bray F, Center MM, Ferlay J, Ward E and Forman D: Global cancer statistics. CA Cancer J Clin 61: 69-90, 2011.

2. Mettlin C, Lee F, Drago J and Murphy GP: The American Cancer Society National Prostate Cancer Detection Project. Findings on the detection of early prostate cancer in 2425 men. Cancer 67: 2949-58, 1991.
3. Nadler RB, Humphrey PA, Smith DS, Catalona WJ and Ratliff TL: Effect of inflammation and benign prostatic hyperplasia on elevated serum prostate specific antigen levels. J Urol 154: 407-413, 1995.

4. Brawer MK: Prostate-specific antigen: Current status. CA Cancer J Clin 49: 264-281, 1999.

5. Gretzer MB and Partin AW: PSA markers in prostate cancer detection. Urol Clin North Am 30: 677-686, 2003.

6. Bartel DP: MicroRNAs: Genomics, biogenesis, mechanism and function. Cell 116: 281-297, 2004.

7. Kloosterman WP and Plasterk RH: The diverse functions of microRNAs in animal development and disease. Dev Cell 11: 441-450, 2006.

8. Ha TY: MicroRNAs in Human diseases: From cancer to cardiovascular disease. Immune Netw 11: 135-154, 2011.

9. Kent OA and Mendell JT: A small piece in the cancer puzzle: MicroRNAs as tumor suppressors and oncogenes. Oncogene 25: 6188-6196, 2006.

10. Lu J, Getz G, Miska EA, Alvarez-Saavedra E, Lamb J, Peck D, Sweet-Cordero A, Ebert BL, Mak RH, Ferrando AA, et al: MicroRNA expression profiles classify human cancers. Nature 435: 834-838, 2005.

11. Ozen M, Creighton CJ, Ozdemir M and Ittmann M: Widespread deregulation of microRNA expression in human prostate cancer. Oncogene 27: 1788-1793, 2008.

12. Dews M, Homayouni A, Yu D, et al: Augmentation of tumor angiogenesis by a Myc activated microRNA cluster. Nat Genet 38: 1060-1065, 2006.

13. Hayashita Y, Osada H, Tatematsu Y, et al: A polycistronic microRNA cluster, miR-17-92, is overexpressed in human lung cancers and enhances cell proliferation. Cancer Res 65: 9628-9632, 2005.

14. Yao Y, Suo AL, Li ZF, et al: MicroRNA profiling of human gastric cancer. Mol Med Rep 2: 963-970, 2009.

15. Ayala de la Peña F, Kanasaki K, Kanasaki M, Tangirala N, Maeda $\mathrm{G}$ and Kalluri R: Loss of p53 and acquisition of angiogenic microRNA profile are insufficient to facilitate progression of bladder urothelial carcinoma in situ to invasive carcinoma. J Biol Chem 286: 20778-20787, 2011.

16. Hsu TI, Hsu CH, Lee KH, et al: MicroRNA-18a is elevated in prostate cancer and promotes tumorigenesis through suppressing STK4 in vitro and in vivo. Oncogenesis 3: e99, 2014.

17. Mitchell PS, Parkin RK, Kroh EM, et al: Circulating microRNAs as stable blood-based markers for cancer detection. Proc Natl Acad Sci USA 105: 10513-10518, 2008.

18. Kosaka N, Iguchi H, Yoshioka Y, Takeshita F, Matsuki Y and Ochiya T: Secretory mechanisms and intercellular transfer of microRNAs in living cells. J Biol Chem 285: 17442-17452, 2010.

19. Zheng H, Zhang L, Zhao Y, et al: Plasma miRNAs as diagnostic and prognostic biomarkers for ovarian cancer. PLoS One 8: e77853, 2013.

20. Chen X, Ba Y, Ma L, et al: Characterization of microRNAs in serum: A novel class of biomarkers for diagnosis of cancer and other diseases. Cell Res 18: 997-1006, 2008.

21. Wang GK, Zhu JQ, Zhang JT, et al: Circulating microRNA: A novel potential biomarker for early diagnosis of acute myocardial infarction in humans. Eur Heart J 31: 659-666, 2010.

22. Roth P, Wischhusen J, Happold C, Chandran PA, Hofer S, Eisele G, Weller M and Keller A: A specific miRNA signature in the peripheral blood of glioblastoma patients. J Neurochem 118: 449-457, 2011.

23. Schrauder MG, Strick R, Schulz-Wendtland R, Strissel PL, Kahmann L, Loehberg CR, Lux MP, Jud SM, Hartmann A, Hein A, et al: Circulating micro-RNAs as potential blood-based markers for early stage breast cancer detection. PLoS One 7: e29770, 2012.

24. Meder B, Keller A, Vogel B, Haas J, Sedaghat-Hamedani F, Kayvanpour E, Just S, Borries A, Rudloff J, Leidinger P, et al: MicroRNA signatures in total peripheral blood as novel biomarkers for acute myocardial infarction. Basic Res Cardiol 106: 13-23, 2011.

25. Keller A, Leidinger P, Bauer A, Elsharawy A, Haas J, Backes C, Wendschlag A, Giese N, Tjaden C, Ott K, et al: Toward the blood-borne miRNome of human diseases. Nat Methods 8: 841-843, 2011.

26. Median-Villaamil V, Martinez-Breijo S, Portela-Pereira P, Quindós-Varela M, Santamarina-Cainzos I, Antón-Aparicio LM and Gómez-Veiga F: Circulating MicroRNAs in blood of patients with prostate cancer. Actas Urol Esp 38: 633-639, 2014 (In English, Spanish). 
27. Gleason DF, Mellinger GT: Prediction of prognosis for prostatic adenocarcinoma by combined histological grading and clinical staging. J Urol 111: 58-64, 1974.

28. Gleason DF: Histologic grading of prostate cancer: A perspective. Hum Pathol 23: 273-279, 1992.

29. Sobin LH and Wittekind CH (eds): TNM Classification of Malignant Tumours, $6^{\text {th }}$ edition. Wiley-Liss, New York, NY, USA, pp 184-187, 2002.

30. Schmittgen TD and Livak KJ: Analyzing real-time PCR data by the comparative C(T) method. Nat Protoc 3: 1101-1108, 2008.

31. Minoo P, Zlobec I, Baker K, Tornillo L, Terracciano L, Jass JP and Lugli A: Prognostic significance of mammalian sterile20-like kinase 1 in colorectal cancer. Mod Pathol 20: 331-338, 2007.

32. Zhou D, Conrad C, Xia F, Park J, Payer B, Yin Y, Lauwers GY, Thasler W, Lee JT, Ayruch J and Bardeesy N: Mst1 and Mst2 maintain hepatocyte quiescence and suppress hepatocellular carcinoma development through inactivation of the Yap1 oncogene. Cancer Cell 16: 425-438, 2009.
33. Morimura R, Komatsu S, Ichikawa D, et al: Novel diagnostic value of circulating miR-18a in plasma of patients with pancreatic cancer. Br J Cancer 105: 1733-1740, 2011.

34. Luo X, Stock C, Burwinkel B and Brenner H: Identification and evaluation of plasma MicroRNAs for early detection of colorectal cancer. PLoS One 8: e62880, 2013.

35. Hirajima S, Komatsu S, Ichikawa D, Takeshita H, Konishi H, Shiozaki A, Morimura R, Tsujiura M, Nagata H, Kawaguchi T, et al: Clinical impact of circulating miR-18a in plasma of patients with oesophageal squamous cell carcinoma. Br J Cancer 108: 1822-1829, 2013.

36. Hoffman RM, Gilliland FD, Adams-Cameron M, Hunt WC and Key CR: Prostate-specific antigen testing accuracy in community practice. BMC Fam Pract 3: 19, 2002. 